\title{
Optimization of tetramycin production in Streptomyces ahygroscopicus S91
}

\author{
Guang Chen(D, Mengqiu Wang, Xianpu Ni and Huanzhang Xia
}

\begin{abstract}
Background: Tetramycin is a 26-member tetraene antibiotic used in agriculture. It has two components, tetramycin A and tetramycin B. Tetramycin B is obtained by the hydroxylation of tetramycin A on C4. This reaction is catalyzed by the cytochrome P450 monooxygenase TtmD. The two components of tetramycin have different antifungal activities against different pathogenic fungi. Therefore, the respective construction of high-yield strains of tetramycin A and tetramycin B is conducive to more targeted action on pathomycete and has a certain practical value.

Results: Streptomyces ahygroscopicus $\mathrm{S} 91$ was used as the original strain to construct tetramycin A high-yield strains by blocking the precursor competitive biosynthetic gene cluster, disrupting tetramycin B biosynthesis, and overexpressing the tetramycin pathway regulator. Eventually, the yield of tetramycin $A$ in the final strain was up to $1090.49 \pm 136.65 \mathrm{mg} \cdot \mathrm{L}^{-1}$. Subsequently, TtmD, which catalyzes the conversion from tetramycin A to tetramycin B, was overexpressed. Strains with 2, 3, and 4 copies of $t \mathrm{tmD}$ were constructed. The three strains had different drops in tetramycin A yield, with increases in tetramycin B. The strain with three copies of $t \mathrm{tmD}$ showed the most significant change in the ratio of the two components.
\end{abstract}

Conclusions: A tetramycin A single-component producing strain was obtained, and the production of tetramycin A increased $236.84 \% \pm 38.96 \%$ compared with the original strain. In addition, the content of tetramycin B in a highyield strain with three copies of $t \mathrm{tmD}$ increased from $26.64 \% \pm 1.97$ to $51.63 \% \pm 2.06 \%$.

Keywords: Tetramycin, Streptomyces ahygroscopicus, Polyene macrolide antibiotics, Cytochrome P450 monooxygenase, Metabolic engineering

\section{Background}

Secondary metabolites produced by microorganisms have various physiological activities and are important sources of natural drugs [1]. In recent years, with the development of biotechnologies, metabolic engineering based on recombinant DNA technology has been increasingly applied to increase the yield of secondary metabolites, bringing advantages in improving the content of metabolic components and producing non-natural new compounds [2]. The primary methods of metabolic engineering are as follows. (1) Changing the distribution

\footnotetext{
* Correspondence: hzxia@syphu.edu.cn
}

School of Life Science and Biopharmaceutics, Shenyang Pharmaceutical University, No.103 Wenhua Road, Shenyang, Liaoning, China of precursor metabolic fluxes: the precursors used to synthesize secondary metabolites are primarily derived from the primary metabolism of microorganisms and are one of the key factors that determine the yield of secondary metabolites. By inactivating the precursor competing biosynthetic gene clusters and changing the metabolic fluxes of the precursor, the yield of the target product can be increased effectively [3-5]. (2) Blocking or overexpressing of the structural genes: Through the inactivation or overexpression of some specific structural genes in the biosynthesis gene cluster (BGC) of secondary metabolites, the metabolic pathway can be terminated at an intermediate product or the metabolite can be further metabolized into a final product to improve

(C) The Author(s). 2021 Open Access This article is licensed under a Creative Commons Attribution 4.0 International License, which permits use, sharing, adaptation, distribution and reproduction in any medium or format, as long as you give appropriate credit to the original author(s) and the source, provide a link to the Creative Commons licence, and indicate if changes were made. The images or other third party material in this article are included in the article's Creative Commons licence, unless indicated otherwise in a credit line to the material. If material is not included in the article's Creative Commons licence and your intended use is not permitted by statutory regulation or exceeds the permitted use, you will need to obtain permission directly from the copyright holder. To view a copy of this licence, visit http://creativecommons.org/licenses/by/4.0/ The Creative Commons Public Domain Dedication waiver (http://creativecommons.org/publicdomain/zero/1.0/) applies to the data made available in this article, unless otherwise stated in a credit line to the data. 
the proportions of the secondary metabolites [6, 7]. (3) Expressing and blocking regulatory genes: The biosynthesis of secondary metabolites is typically regulated by specific regulators within its BGC, and sometimes by a pleiotropic regulator. Pathway-specific regulatory factors can activate or inhibit the transcription of structural genes. Overexpression of positive regulators or inhibition of negative regulators can be beneficial for the accumulation of secondary metabolites [8-11]. In addition, (i) increasing the resistance, (ii) the use of genome rearrangement technology, and (iii) using heterologous hosts or the expression of BGCs in production strains [12] are also common methods of metabolic engineering.

Polyene macrolides are polyketones that include pimaricin, nystatin, amphotericin, and tetramycin. Polyene macrolides are primarily used in the treatment of clinical fungal infections, food preservation, and agricultural fungal disease prevention. In addition, polyene macrolides show antitumor, antiviral, antiprotozoal and immunosuppressive activities with great antibacterial potential [13-16]. Tetramycin is a 26-member tetraene macrolide that can be produced by Streptomyces noursei, Streptomyces hygrospinosus, and Streptomyces ahygroscopicus [17]. As a biological fungicide, tetramycin is primarily used for the prevention and control of leaf spot disease, rice blast disease, and gray mold [18-20]. Tetramycin consists of two components, tetramycin A (TA) and tetramycin $\mathrm{B}$ (TB). However, the antifungal activities of the two components are different. TA prefers to prevent Saccharomyces cerevisiae and Aspergillus flavus, whereas TB has stronger effects on Fusarium solani, Penicillium notatum, and Scopulariopsis [21, 22]. Therefore, it is of potential value to obtain high-yield strains of each of the two components for industrial production.

The biosynthetic pathway and regulatory factors of tetramycin have been studied [23-25]. Tetramycin biosynthesis follows the common polyene antibiotics biosynthesis pathway, with small molecular carboxylic acids (acetyl-CoA, malonyl-CoA, methylmalonyl-CoA, and ethylmalonyl-CoA) as TA precursors. Tetramycinolide is formed by the polyketide synthase pathway (PKS), TA is formed through carboxylation and glycosylation by post-PKS tailoring, and $\mathrm{TB}$ is formed from TA through C4 hydroxylation (Fig. S1). In this study, the production of tetramycin is improved by means of metabolic engineering using Streptomyces ahygroscopicus S91(GCMCC 4.7082) as the original strain. The genome of S. ahygroscopicus S91 contains various BGCs and produces several of secondary metabolites, including tetramycin, nystatin, anisomycin, and toyocamycin. Tetramycin and nystatin are polyene macrolide antibiotics that share the common precursors acetyl-CoA,
malonyl-CoA, and methylmalonyl-CoA in the biosynthesis process. By blocking the biosynthesis of nystatin, the metabolic fluxes of the precursors are redirected, improving the production of tetramycin. TB is the hydroxylation product of TA, which is converted by the cytochrome $\mathrm{P} 450$ monooxygenase TtmD. Blocking ttmD expression can eliminate the conversion to TB. Hence, TA is obtained separately. The overexpression of $t t m \mathrm{D}$ is beneficial to increase the conversion efficiency of TA, and the proportion of TB can be raised. Cui et al. conducted a study on the regulatory mechanism of tetramycin biosynthesis and found that there were four pathway-specific regulators (TtmRI, TtmRII, TtmRIII, and TtmRIV) in the tetramycin BGC. TtmRIV belongs to the positive regulatory factors of the PAS-LuxR family, and its overexpression improved the TA yield [25].

By using the metabolic engineering for the biosynthesis of secondary metabolites, the tetramycinproducing strain, S. ahygroscopicus S91, is expected to optimize the composition of tetramycin and enrich the metabolic engineering application of polyene macrolide antibiotics.

\section{Results}

\section{Construction of the tetramycin-producing strain by disrupting the biosynthesis of nystatin}

The primary intracellular fermentation products of $S$. ahygroscopicus $\mathrm{S} 91$ are tetramycin (TA and TB) and nystatin (NA1) (Fig. S2). Acetyl-CoA, malonyl-CoA, and methylmalonyl-CoA were the precursors used in the biosynthesis process of these products. Therefore, an increase in the yield of tetramycin was expected by disrupting the biosynthesis of nystatin and redirecting the fluxes of precursors to the biosynthetic pathway of tetramycin. $N y s B$, the first extension module of PKS in nystatin BGC, was chosen to be disrupted to minimize the loss of precursors. A $1714 \mathrm{bp}$ DNA fragment in the nys B framework was deleted to construct the nystatin disruption strain S91- $\triangle \mathrm{NB}$ (Fig. 1a, Fig. S3). The fermentation results of $S 91-\triangle N B$ showed that $S 91-\Delta N B$ no longer produced NA1. In addition, the TA and TB contents increased from $35.61 \% \pm 1.80$ and $21.31 \% \pm 1.18$ to $51.59 \% \pm 3.90$ and $26.64 \% \pm 1.97 \%$, respectively. The total production of TA and TB increased from $517.49 \pm 24.72$ $\mathrm{mg} \cdot \mathrm{L}^{-1}$ to $657.25 \pm 29.77 \mathrm{mg} \cdot \mathrm{L}^{-1}$, respectively (Fig. 1e, f, Tab. S1).

\section{Construction of the TA single-component high-yield strain}

TA and TB have different antifungal effects on different fungi, and TA has stronger antifungal effects on Saccharomyces cerevisiae and Aspergillus flavus than TB. TB is hydroxylated TA, and the reaction is catalyzed using the cytochrome P450 monooxygenase TtmD. A TA single- 


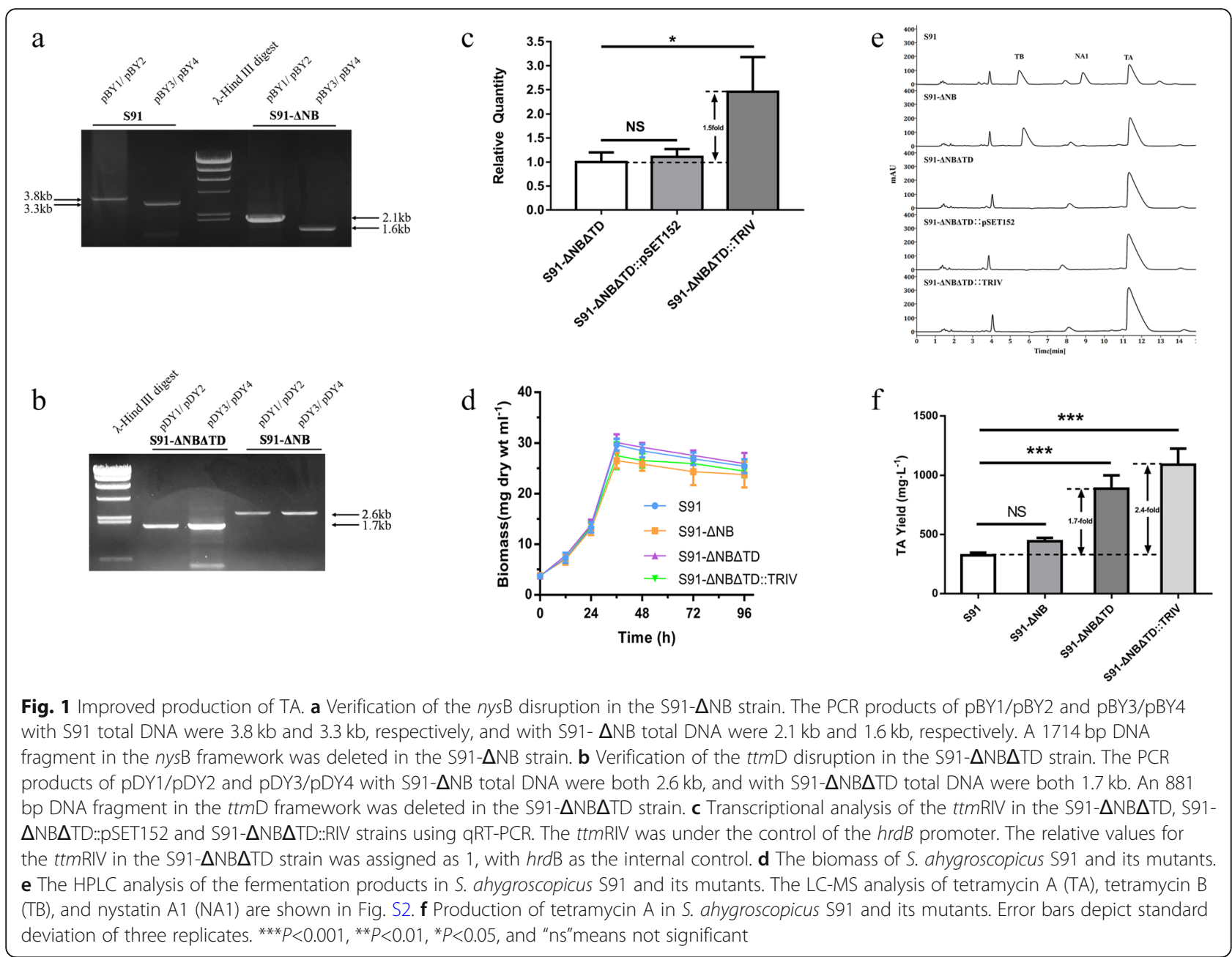

component strain was expected to be obtaind by blocking the ttmD. TtmD is located upstream of the PKS ttmS1, and the two genes have opposite polarities. To avoid influences on the $t$ tmS 1 transcription, an $881 \mathrm{bp}$ fragment within the framework of $t$ tm $\mathrm{D}$ was deleted to generate S91- $\triangle \mathrm{NB} \Delta \mathrm{TD}$ (Fig. 1b, Fig. S4). The recombinant strain S91- $\triangle \mathrm{NB} \Delta \mathrm{TD}$ produced only TA. The total yield of tetramycin in the S91- $\triangle \mathrm{NB} \Delta \mathrm{TD}$ strain was $33.18 \% \pm 12.16 \%$ higher than that of S91- $\triangle \mathrm{NB}$. The yield of TA increased significantly, from $443.22 \pm 29.19$ $\mathrm{mg} \cdot \mathrm{L}^{-1}$ to $888.62 \pm 111.98 \mathrm{mg} \cdot \mathrm{L}^{-1}$ (an increase of $100.49 \% \pm 15.57 \%$ ) (Fig. 1e, f, Tab. S1).

Previous work demonstrated that $t$ tmRIV was a positive regulatory gene in tetramycin $\mathrm{BGC}$, and its overexpression improved the TA yield. Therefore, the addition of the copy of ttmRIV in the TA single-component producing strain was expected to further improve the TA yield. Using the $S$. ahygroscopicus S91 total DNA as a template, the $624 \mathrm{bp}$ ttmRIV fragment was obtained by PCR and inserted into pSET152 to construct an overexpression plasmid. The ttmRIV was under the control of the $h r d B$ promoter. Then $S 91-\triangle N B \Delta T D$ strain was transformed to obtain S91- $\triangle \mathrm{NB} \Delta \mathrm{TD}: \mathrm{TRIV}$ and S91$\triangle \mathrm{NB} \triangle \mathrm{TD}:: \mathrm{pSET} 152$. The qRT-PCR analysis showed that the expression level of ttmRIV in the overexpression strain S91- $\triangle \mathrm{NB} \Delta \mathrm{TD}:$ :TRIV was nearly 1.5 times higher than that in S91- $\triangle \mathrm{NB} \Delta \mathrm{TD}$ (Fig. 1c). The fermentation results showed that the TA yield in S91- $\triangle \mathrm{NB} \Delta \mathrm{TD}$ ::TRIV reached $1090.49 \pm 136.65 \mathrm{mg} \cdot \mathrm{L}^{-1}$, which was $22.75 \% \pm$ $2.40 \%$ higher than that in S91- $\triangle \mathrm{NB} \Delta \mathrm{TD}$ (Fig. 1e, f, Tab. S1). The biomass of $S$. ahygroscopicus S91 and its mutants was shown in Fig. 1d.

\section{Construction of the TB high-yield strains by using the overexpression of $t t m D$}

$\mathrm{TB}$ is produced by the hydroxylation of TA on $\mathrm{C} 4$, which is catalyzed by the cytochrome P450 monooxygenase TtmD. Therefore, it was hypothesized that the production of $\mathrm{TB}$ could be improved by increasing the copies of $t t m D$ in the S91- $\Delta \mathrm{NB}$ strain. Single-copy, twocopy, and three-copy ttmD plasmids were constructed based on the pSET152 vector, and three multicopy 
plasmids and pSET152 were introduced into the S91$\triangle \mathrm{NB}$ strain, S91- $\mathrm{NB}:: \mathrm{TD}, \mathrm{S} 91-\Delta \mathrm{NB}:: 2 \mathrm{TD}$, and S91$\triangle \mathrm{NB}:: 3 T D$, and S91- $\triangle \mathrm{NB}:: \mathrm{pSET} 152$ strains were obtained. The $t t m \mathrm{D}$ was also under the control of the $h r d B$ promoter. The expression of $t$ tm $\mathrm{D}$ in the multicopy ttmD strains was analyzed using qRT-PCR. Compared with the original strain, the expression levels of ttmD in the recombinant strains increased 10.3-, 29.0-, and 18.9fold (Fig. 2b).

Subsequently, the tetramycin content in the recombinant strains during the fermentation period were observed (Fig. 2c). The total tetramycin content was slightly lower within $24 \mathrm{~h}$, and each strain ranged from 64 to $69 \%$. As the fermentation time increased, the tetramycin yield increased and the content increased slightly. After $48 \mathrm{~h}$, the content of the two components in each strain was within the range of $73-77 \%$. The TA content of S91- $\triangle \mathrm{NB}$ was basically stable at each fermentation time, while the TB content increased slightly $(6.69 \% \pm$ $0.43 \%$ ). The proportion of TA from $24 \mathrm{~h}$ to $96 \mathrm{~h}$ decreased by $6.81 \% \pm 1.01,7.36 \% \pm 0.27$, and $5.78 \% \pm 0.56 \%$ in the multicopy $t$ tmD recombinant strains. TB showed an increasing trend, and the proportion of TB at $96 \mathrm{~h}$ was $14.04 \% \pm 0.92,13.20 \% \pm 0.73$, and $15.56 \% \pm 0.77 \%$ higher. The proportion of TB increased with the proportion of TA dropped, which indicated that TA gradually transformed into $\mathrm{TB}$ during the fermentation process. The TB content in S91- $\triangle \mathrm{NB}:: 2 \mathrm{TD}$ increased to $51.63 \% \pm 2.06 \%$, and the yield reached $370.79 \pm 14.79$ $\mathrm{mg} \cdot \mathrm{L}^{-1}$, nearly twice as high as in the original strain. The TB content in S91- $\triangle \mathrm{NB}:: 3 \mathrm{TD}$ increased to $44.76 \% \pm 1.90 \%$, and the yield reached $533.59 \pm 22.65$ mg. $\mathrm{L}^{-1}$ (Fig. 2d, Tab. S1). The biomass of S91- $\Delta \mathrm{NB}$ and its mutants was shown in Fig. 2a.

\section{Discussion}

Tetramycin is an important fungicide, and its strains are known to be associated with the production of other polyene macrolide antibiotics, such as nystatins and tetrins [22]. It is difficult to separate tetramycin from the by-products of its producing strains owing to their similar synthesis pathways and common precursors. Metabolic engineering is an effective and practical approach to reducing the quantity of by-products and improving the yield of tetramycin. In this study, the competing pathway of nystatin biosynthesis was blocked in the original strain S. ahygroscopicus S91, mainly generating the tetramycin strain S91- $\triangle \mathrm{NB}$. Relative to the original strain, S91- $\triangle \mathrm{NB}$ showed no significant improvement in the total yield of tetramycin, as the reduction in nystatin was almost equivalent to the increase in tetramycin. However, in the subsequent experiment, when the cytochrome P450 monooxygenase TtmD was inactive in the recombinant strain $\mathrm{S} 91-\triangle \mathrm{NB} \Delta \mathrm{TD}$, the total yield of tetramycin increased $33.18 \% \pm 12.16 \%$ over $S 91-\Delta \mathrm{NB}$. $\mathrm{TtmD}$ is responsible for the conversion from TA to TB, which is similar to its counterpart NysL, AmphL, and PimD, performing the final biosynthetic step on polyene. These four enzymes catalyze different chemical reactions: hydroxylation of the $\mathrm{C} 5$ in tetramycin (TtmD), hydroxylation of the $\mathrm{C} 10$ in nystatine (NysL) [26], hydroxylation of the $\mathrm{C} 8$ in amphotericin (AmphL) [27], and epoxidation of the C4-C5 double bond in pimaricin (PimD) [28]. All of these reactions require NADPH as a reducing factor. In the biosynthesis of polyenes and other polyketides, NADPH is usually consumed in the reduction of enoylreductase (ER) of PKS and the tailoring modification of macrolides [29,30]. Disruption of $t t m \mathrm{D}$ in S91- $\triangle \mathrm{NB} \Delta \mathrm{TD}$ decreased NADPH consumption, and more NADPH was redirected into biosythesis of PKS to improve the yield of TA to some extent. For the same reason, an excessive overexpression of $t$ tmD may weaken the biosynthesis of PKS. Even though the proportion of TA and TB showed the greatest optimization in the three-copy $t t m \mathrm{D}$ strain S91- $\triangle \mathrm{NB}:: 2 \mathrm{TD}$, the total yield of tetramycin was not the highest.

Regarding the overexpression of $t$ tmRIV and $t t m \mathrm{D}$, the $h r d B$ promoter was used to control the transcription. Generally, the introduction of a strong promoter is an effective strategy for improving product yield and activating cryptic gene clusters [31]. In our previous study on $t t m \mathrm{D}$, three promoters, including the $t t m \mathrm{D}$ native promoter, the ermE* promoter, and the $h r d B$ promoter, were separately introduced into the $t t m \mathrm{D}$ disruption strain S91- $\triangle \mathrm{TD}$ and the efficiency of expression was assessed. We found the $h r d B$ promoter to be the most efficient, and this was confirmed in the multicopy $t$ tm $\mathrm{D}$ strains. Regarding ttmRIV, the $h r d B$ promoter fostered efficiency to a much lower extent than $t$ tmD, so the improvement in the yield of TA was limited. Currently, stronger promoters, such as $k a s O \mathrm{p}^{*}$ are used to overexpress the rate-limiting biosynthetic genes in some streptomyces, and the yield of products improved significantly [32, 33]. In this way, this method offers the opportunity to further improve the TA yield by overexpression of $t$ tmRIV under these promoters and by introducing multiple copies of $t$ tmRIV.

Many other metabolic engineering approaches can also improve the yield of both TA and TB. In these strategies, increasing the supply of precursors can be direct and effective. Generally, the supply of various acyl-CoAs is the limiting factor in the biosynthesis of polyketides. It can be overcome by overexpressing the genes encoding the key enzymes such as acetyl-CoA carboxylase (ACC), propionyl-CoA carboxylase (PCC), and crotonyl-CoA carboxylase/reductase (CCR) [34-36]. ACC catalyzes the conversion from acetyl-CoA to malnonyl-CoA, PCC plays a key role in increasing methylmalonyl-CoA, and 


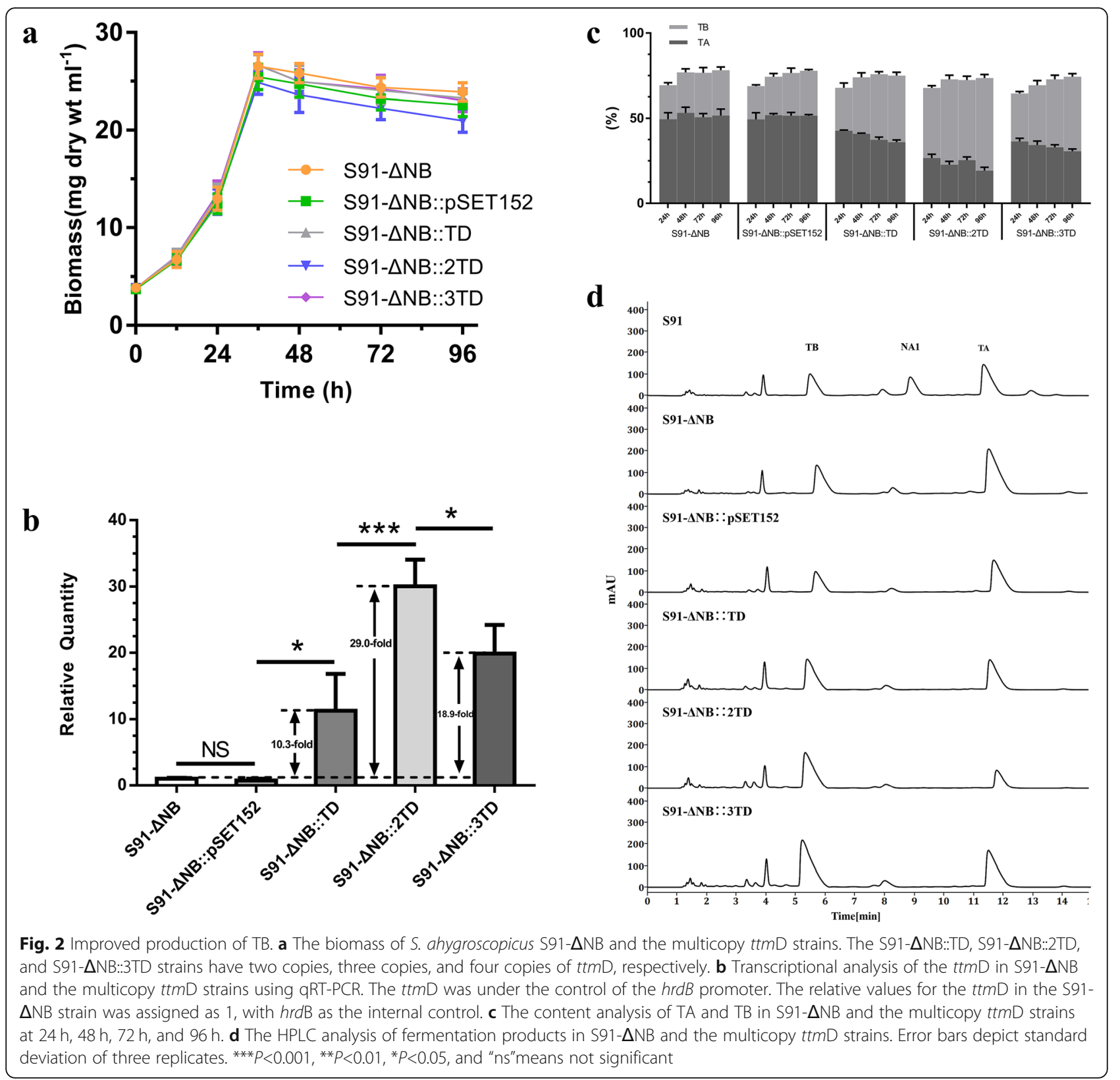

CCR catalyzes the conversion from crotonyl-CoA to ethylmalonyl-CoA. Ten malonyl-CoAs, one methylmalonyl-CoA, and one ethylmalonyl-CoA are involved in the biosynthesis of tetramycin, so the overexpression of these genes is expected to increase the intracellular pool of precursors and improve the yield of tetramycin. Recently, branched chain amino acid (BCAA) metabolism has been shown to be the bottleneck of the biosynthesis of some polyketides [37, 38]. BCAA degradation pathway can supply various acyl-CoAs. Manipulation on the key enzymes of BCAA metabolism, such as branched chain $\alpha$-keto acid dehudrogenase (BCDH) and acyl-CoA dehydrogenase (ACAD), can also improve the yield of tetramycin.

As mentioned above, cytochrome P450 monooxygenases catalyze hydroxylation (TtmD, NysL, and AmphL) or epoxidation (PimD) of polyene macrolides. TtmD shows $59 \%$ sequence identity with NysL, $56 \%$ with AmphL and 68\% with PimD. It has been reported [39] that these enzymes are not as substrate-specific as previously thought. TtmD, nysL, and $a m p h \mathrm{~L}$ were separately introduced into a Streptomyces natalensis $\Delta$ pimD strain whose fermentation product was 4,5-desepoxypimamycin (DEP). The results showed that TtmD can convert 
DEP into pimaricin, indicating that TtmD can perform hydroxylation and epoxidation. NysL catalyzes the conversion of DEP to C6-OH-DEP, a new derivative. However, AmphL does not catalyze the conversion. A similar experiment was performed in S91- $\Delta$ NB $\Delta$ TD. Phylogenetic tree analysis and homology modeling results show that TtmD and PimD have considerable structural similarity. For this reason, pimD was introduced into S91$\triangle \mathrm{NB} \triangle \mathrm{TD}$ as $t \mathrm{tmD}$. Although PimD was expressed in S91- $\triangle \mathrm{NB} \Delta \mathrm{TD}$, TA was not converted to TB. Furthermore, no other new products were detected (not shown). These results indicate that the substrate specificity of PimD is more strict than that of TtmD. PimD recognizes double bond at C4-C5 of DEP, whereas the counterpart of TA is a saturated bond. The saturated bond is caused by the inactivation DH11 in PKS of tetramycin BGC. It is reasonable to assume that domain swapping, by some active agents such as DH11 in PKS of pimaricin BGC, could generate a double bond at $\mathrm{C} 4-\mathrm{C} 5$ which is subsequently epoxidized by TtmD to form new tetramycin derivatives.

\section{Conclusions}

High-yield strains that produced TA and TB were obtained in this study. During the process of constructing the high yield strain of TA, nystatin BGC was first blocked, and yield of tetramycin was increased to $667.25 \pm 29.77 \mathrm{mg} \cdot \mathrm{L}^{-1}$; hence, the TA yield was $443.22 \pm$ $29.19 \mathrm{mg} \cdot \mathrm{L}^{-1}$. Then, ttmD was blocked, the TA yield was increased to $888.62 \pm 111.98 \mathrm{mg} \cdot \mathrm{L}^{-1}$. After that, the positive regulatory gene of tetramycin $\mathrm{BGC}$ was overexpressed. Hence, the TA yield was further increased to $1090.49 \pm 136.65 \mathrm{mg} \cdot \mathrm{L}^{-1}$.

In the construction of $\mathrm{TB}$ high-proportion strain, strains with different copy numbers of $t t m \mathrm{D}$ were constructed to increase the content of $\mathrm{TB}$. The results showed that the TB content in the strain with three copies of $t \mathrm{tmD}$ was the highest, increasing from $26.64 \% \pm$ 1.97 to $51.63 \% \pm 2.06 \%$.

\section{Methods}

\section{Strains, plasmids, medium, and cultivation conditions}

The strain S. ahygroscopicus S91 was used as the initial strain, which had been deposited at the China General Microbiology Culture Collection Center (accession No. CGMCC 4.7082), Institute of Microbiology, the Chinese Academy of Science. The other plasmids and primers used in this study are listed in Table S2.

S. ahygroscopicus S91 and its mutants were maintained on Gause's synthetic agar medium (2\% soluble starch, $0.1 \%$ Beef extract, $0.1 \% \mathrm{KNO}_{3}, 0.05 \% \mathrm{MgSO}_{4} \cdot 7 \mathrm{H}_{2} \mathrm{O}$, $0.05 \% \mathrm{~K}_{2} \mathrm{HPO}_{4} \cdot 3 \mathrm{H}_{2} \mathrm{O}, 0.05 \% \mathrm{NaCl}, 0.001 \% \mathrm{FeSO}_{4} \cdot 7 \mathrm{H}_{2} \mathrm{O}$, $2.5 \%$ agar, and $\mathrm{pH} 7.2$ ) at $28^{\circ} \mathrm{C}$. E. coli strains were cultured in the LB broth or agar at $37^{\circ} \mathrm{C} .2 \times \mathrm{YT}$ medium was used for the germination of Streptomyces spores, and MS was used for co-culture of Streptomyces and $E \cdot c o l i$ in conjugation. For fermentation, the seed medium contained $2 \%$ glucose, $0.6 \%$ peptone, $0.6 \%$ yeast extract, $1 \% \mathrm{NaCl}$, at a $\mathrm{pH}$ of 7.2. The fermentation medium contained $2 \%$ corn powder, $0.8 \%$ corn starch, $3 \%$ soya bean, $3 \%$ glucose, $0.02 \% \mathrm{NaCl}, 0.02 \% \mathrm{MgSO}_{4} \cdot 7 \mathrm{H}_{2} \mathrm{O}, 0.02 \%$ $\mathrm{K}_{2} \mathrm{HPO}_{4} \cdot 3 \mathrm{H}_{2} \mathrm{O}, 0.02 \% \quad \mathrm{FeSO}_{4} \cdot 7 \mathrm{H}_{2} \mathrm{O}, 0.25 \%\left(\mathrm{NH}_{4}\right)_{2} \mathrm{SO}_{4}$, $0.5 \% \mathrm{CaCO}_{3}$, at a pH of 7.2 . Then $2 \%$ agar was added to this for the solid fermentation medium used to culture the mycelia during the process of RNA extraction.

\section{Inactivation of nysB}

To disrupt the biosynthesis of nystatin, the genomic DNA of S. ahygroscopicus S91 was used as a template, and the primers NB-UF/NB-UR and NB-DF/NB-DR were used for the PCR. The $1452 \mathrm{bp}$ upstream homologous fragment, $N B U$, and the 1456 bp downstream homologous fragment, $N B D$, were obtained using PCR amplification. After sequencing verification, they were jointly ligated to the pKC1139 vector between the HindIII and BamHI restriction sites, and the blocking plasmid pDNB was constructed (Fig. S3a). After that, pDNB was transferred into E. coli ET12567 (pUZ8002) and introduced into S. ahygroscopicus S91 by conjugation, and apramycin-resistant strains were selected for subculture. The stable apramycin-sensitive strains were screened after three generations of relaxed culture. The nystatin disruption strain, S91- $\triangle \mathrm{NB}$, was obtained. Two validation primer pairs (pBY1/pBY2 and $\mathrm{pBY} 3 / \mathrm{pBY} 4)$ were used for the double crossover validation using PCR amplification (Fig. S3b, c).

\section{Inactivation of $t$ tmD}

The primers TD-UF/TD-UR and TD-DF/TD-DR were used to amplify the $1538 \mathrm{bp}$ upstream homologous fragment, $T D U$, and the $1005 \mathrm{bp}$ downstream homologous fragment, $T D D$, of $t t m \mathrm{D}$. After sequencing verification, they were jointly ligated to the pKC1139 vector between the HindIII and EcoRI restriction sites, and the blocking plasmid, pDTD, was constructed (Fig. S4a). After that, pDTD was transferred into E. coli ET12567 (pUZ8002) and introduced into S. ahygroscopicus S91- $\triangle \mathrm{NB}$ by conjugation. The apramycin-resistant strains were selected for subculture, and the stable apramycin-sensitive strains were screened after three generations of relaxed culture. The $t$ tmD deletion strain, S91- $\Delta \mathrm{NB} \Delta \mathrm{TD}$, was then obtained. Two validation primer pairs (pDY1/pDY2 and $\mathrm{pDY} 3 / \mathrm{pDY} 4)$ were used for the double crossover validation using PCR amplification (Fig. S4b, c).

\section{Cloning and overexpression of ttmRIV}

The primers, TRIV-F and TRIV-R, were used to amplify the $624 \mathrm{bp} t$ tmRIV gene fragment. The ttmRIV fragment 
was digested using $N c o \mathrm{I}$ and $\mathrm{XhoI}$ and ligated to pPT2925, which was digested using the same enzymes, to generate the recombinant plasmid pTRIV. pTRIV was digested using $B g l \mathrm{II}$ and a $1.5 \mathrm{~kb}$ fragment containing the $h r d B$ promoter, $t$ tmRIV, and the $T_{0}$ terminator was ligated to pSET152. pSET152 was digested using BamHI and dephosphorylated to construct overexpression plasmid pETRIV (Fig. S5a). After this, pETRIV was transferred into E. coli ET12567 (pUZ8002) and introduced into $S$. ahygroscopicus $S 91-\triangle \mathrm{NB} \Delta \mathrm{TD}$ by conjugation, and the apramycin-resistant strains were selected. The strain S91- $\triangle \mathrm{NB} \Delta \mathrm{TD}:$ :TRIV with two copies ttmRIV was obtained. The primers, PB-1/TRIV-R, were used for verification (Fig. S5b).

\section{Cloning and overexpression of ttmD}

The $1191 \mathrm{bp} t t m \mathrm{D}$ fragment, $T D$, was amplified from $S$. ahygroscopicus S91 genomic DNA using the primers TD-F and TD-R and ligated to the plasmid PPT2925, digested using $\mathrm{NcoI}$ and $\mathrm{XhoI}$, to generate the recombinant plasmid pPTD. The $2.1 \mathrm{~kb}$ fragment containing the $h r d B$ promoter, $T D$, and the $T_{O}$ terminator $\left(P_{h r d B}-T D\right.$ $\left.T_{0}\right)$ was obtained using BglII digestion and ligated to pSET152, which was digested with BamHI and dephosphorylated to construct the overexpression plasmid pETD.

The $2.1 \mathrm{~kb} P_{h r d B}-T D-T_{O}$ fragment was obtained when pPTD was digested using EcoRI and SpeI, and then ligated to the pPTD between the EcoRI and XbaI restriction sites to generate the recombinant plasmid p2PTD. Then the p2PTD was digested using BglII, and the 4.2 kb fragment containing two copies of $P_{h r d B}-T D-T_{O}$ was ligated to pSET152, which was digested using BamHI and dephosphorylated to construct the overexpression plasmid p2ETD, which contained two copies of ttmD (Fig. S6a).

The construction of p3PTD with three copies of $t$ tmD was similar to the construction of p2PTD in which the $2.1 \mathrm{~kb} P_{\text {hrdB }}-T D-T_{O}$ fragment was ligated to p2PTD. In addition, the overexpression plasmid p3ETD was obtained from P3PTD in the same manner as P2ETD as described above.

All of the three plasmids pETD, p2ETD, and p3ETD were transferred into E. coli ET12567 (pUZ8002) and introduced into $S$. ahygroscopicus S91- $\triangle \mathrm{NB}$ by conjugation, and the apramycin-resistant strains were selected. Three multicopy ttmD strains were obtained. The primers, PB-1/TD-R, were used for verification (Fig. S6b).

\section{Purification of tetramycin and detection conditions using HPLC}

S. ahygroscopicus $\mathrm{S} 91$ and its mutants were cultured in seed medium at $28^{\circ} \mathrm{C}$ for $24 \mathrm{~h}$. The seed cultures were inoculated into fermentation medium to $10 \%(\mathrm{v} / \mathrm{v})$ and cultured at $28{ }^{\circ} \mathrm{C}$ for $96 \mathrm{~h}$. Then the mycelia was harvested and extracted with methanol.

The extracts were subjected to HPLC analysis (Agilent series 1260, Agilent Technologies, USA) under the following conditions: column: Agilent EC-C18 column $(150 \times 4.6 \mathrm{~mm}, 4 \mu \mathrm{m})$; column temperature: $34^{\circ} \mathrm{C}$; wave length: $304 \mathrm{~nm}$; flow rate: $1 \mathrm{~mL} \cdot \mathrm{min}^{-1}$; injection volume: $5 \mu \mathrm{L}$; mobile phase: water (solvent A) and methanol: formic acid $=60: 0.1$ (solvent $B$ ). Elution was performed as follows: $40 \%$ A: $60 \%$ B, $0-5 \mathrm{~m}$; down to $35 \%$ A: $65 \%$ B, 5-8 m; 35\% A: 65\%, B 8-18 m.

\section{RNA isolation and the qRT-PCR analysis}

S. ahygroscopicus S91 and its mutants were cultured on a solid fermentation medium for $48 \mathrm{~h}$. Then the mycelia was harvested, and the total RNA were isolated using the Ultrapure RNA Kit (DNase I) (Cwbio). cDNA was reverse transcripted using the PrimeScript ${ }^{\mathrm{m}} \mathrm{RT}$ Reagent Kit (TaKaRa). The qRT-PCR analysis was performed using the MightyAmp ${ }^{\mathrm{mm}}$ for Real Time (SYBR ${ }^{\circ}$ Plus) (TaKaRa). The relative mRNA levels were analyzed using the $2^{-\Delta \Delta \mathrm{Ct}}$ method, with the housekeeping gene $h r d \mathrm{~B}$ as an internal reference. The $h r d \mathrm{~B}$ was amplified using the primers PB-RT-1 and PB-RT-2. The ttmRIV was amplified using the primers RIV-RT-1 and RIV-RT-2. The $t$ tmD was amplified using the primers TD-RT-1 and TD-RT-2.

\section{Abbreviations}

PKS: Polyketide synthase; TA: Tetramycin A; TB: Tetramycin B; NA1: Nystatin; BGCs: Biosynthetic gene clusters

\section{Supplementary Information}

The online version contains supplementary material available at https://doi. org/10.1186/s13036-021-00267-4.

Additional file 1: Figure S1. Biosynthesis of tetramycin.

Additional file 2: Figure S2. LC-MS analysis of tetramycin and nystatin in Streptomyces ahygroscopicus S91. a. The HPLC profile of Streptomyces ahygroscopicus $\mathrm{S} 91$ fermentation products; $b$. The UV absorption spectra and MS of tetramycin B; C. The UV absorption spectra and MS of nystatin; d. The UV absorption spectra and MS of tetramycin A.

Additional file 3: Figure S3. Inactivation of nysB in S.ahygroscopicus S91. a. Construction of the recombinant plamid pDNB; b. Double crossover validation of the recombinant strain $S 91-\Delta N B$; c.Verification of sequencing in the recombinant strain S91- $\Delta N B$.

Additional file 4: Figure S4. Inactivation of ttmD in S.ahygroscopicus S91- $\Delta N B$. a. Construction of the recombinant plasmid pDTD; b. Double crossover validation of the recombinant strain S91- $\triangle N B \Delta T D ; C$. Verification of sequencing in the recombinant strain $591-\triangle N B \Delta T D$.

Additional file 5: Figure S5. Cloning and overexpression of ttmRIV. a. Construction of the recombinant plamid pETRIV; $b$. PCR analysis of the recombinant strain S91- $\triangle$ NB $\Delta$ TD::TRIV, M. DL2000 (2.0k, 1.0k, 0.75k, 0.5k, 0.25k, 0.1k), 1. S91- $\Delta$ NB $\Delta$ TD::pETRIV/PB-1\& TRIV-R (1.1k), 2. S91- $\Delta$ NB $\Delta T D /$ PB-1\&TRIV-R, 3. S91- $\Delta$ NB $\Delta$ TD::pSET152/PB-1\&TRIV-R.

Additional file 6: Figure S6. Cloning and overexpression of ttmD. a. Construction of the recombinant plamid P2ETD; b. PCR analysis of the multicope ttmD recombinant strains, M. DL2000, 1. S91- $\triangle$ NB:.pETD/PB- 
1\&TD-R (1.7k), 2. S91- $\triangle N B:: p 2 E T D / P B-1 \& T D-R ~(1.7 k), 3.591-\Delta N B:: p 3 E T D / P B-$ 1\&TD-R (1.7k), 4. S91- $\triangle N B / P B-1 \& T D-R, 5 . ~ S 91-\triangle N B:: p S E T 152 / P B-1 \& T D-R$

Additional file 7: Table S1. Production analysis in S.ahygroscopicus $S 91$ and its mutants.

Additional file 8: Table S2. Strains, plasmids and primers used in this study.

\section{Acknowledgements}

Not applicable.

\section{Authors' contributions}

HzXia supervised this study. HzXia and GChen conceived and designed the experiments. All authors analyzed the data and wrote the manuscript. All authors read and approved the final manuscript.

\section{Funding}

The project for Disciplinary leaders of universities supporting the Northwest Economic and Social Development of Liaoning Province (10163-1007-2). The Scientific Research Fund of Liaoning Provincial Education Department (2017LZD05, 2019LZD03).

\section{Availability of data and materials}

The DNA sequence of the tetramycin BGC is available to the public in NCB under accession number: JX827252.1.

\section{Declarations}

Ethics approval and consent to participate

Not applicable.

\section{Consent for publication}

Not applicable.

\section{Competing interests}

The authors declare that they have no competing interests.

Received: 18 February 2021 Accepted: 7 May 2021

Published online: 22 May 2021

\section{References}

1. Katz L, Baltz R. Natural product discovery: past, present, and future. J Ind Microbiol Biotechnol. 2016;43(2-3):155-76. https://doi.org/10.1007/s10295015-1723-5.

2. Weber T, Charusanti $P$, Musiol-Kroll E, Jiang $X$, Tong $Y$, Kim H, et al. Metabolic engineering of antibiotic factories: new tools for antibiotic production in actinomycetes. Trends Biotechnol. 2015;33(1):15-26. https:// doi.org/10.1016/j.tibtech.2014.10.009.

3. Wei $X$, Yunxiang L, Yinghua Z. Enhancement and selective production of Oligomycin through inactivation of Avermectin's starter unit in Streptomyces avermitilis. Biotechnol Lett. 2006;28(12):911-6. https://doi.org/10.1007/s1052 9-006-9012-z.

4. Huang D, Xia M, Li S, Wen J, Jia X. Enhancement of FK506 production by engineering secondary pathways of Streptomyces tsukubaensis and exogenous feeding strategies. J Ind Microbiol Biotechnol. 2013;40(9):102337. https://doi.org/10.1007/s10295-013-1301-7.

5. Gao W, Wu Z, Sun J, Ni X, Xia H. Modulation of kanamycin B and kanamycin a biosynthesis in Streptomyces kanamyceticus via metabolic engineering. PLoS One. 2017;12(7):e0181971. https://doi.org/10.1371/journal.pone.0181 971.

6. Ikeda H, Wang L, Ohta T, Inokoshi J, Ōmura S. Cloning of the gene encoding avermectin B 5-O-methyltransferase in avermectin-producing Streptomyces avermitilis. Gene. 1998;206(2):175-80. https://doi.org/10.1016/ S0378-1119(97)00581-7.

7. Chen Y, Deng W, Wu J, Qian J, Chu J, Zhuang Y, et al. Genetic modulation of the overexpression of tailoring genes eryK and eryG leading to the improvement of erythromycin a purity and production in Saccharopolyspora erythraea fermentation. Appl Environ Microbiol. 2008;74(6):1820-8. https:// doi.org/10.1128/AEM.02770-07.
8. Stratigopoulos G, Bate N, Cundliffe E. Positive control of tylosin biosynthesis: pivotal role of TyIR. Mol Microbiol. 2004;54(5):1326-34. https://doi.org/1 0.1111/j.1365-2958.2004.04347.x.

9. Antón N, Santos-Aberturas J, Mendes M, Guerra S, Martín J, Aparicio J. PimM, a PAS domain positive regulator of pimaricin biosynthesis in Streptomyces natalensis. Microbiology. 2007;153(9):3174-83. https://doi.org/1 0.1099/mic.0.2007/009126-0.

10. Wu H, Liu W, Dong D, Li J, Zhang D, Lu C. SInM gene overexpression with different promoters on natamycin production in Streptomyces lydicus A02. J Ind Microbiol Biotechnol. 2013;41(1):163-72.

11. Baltz R. Genetic manipulation of secondary metabolite biosynthesis for improved production in Streptomyces and other actinomycetes. J Ind Microbiol Biotechnol. 2015;43(2-3):343-70. https://doi.org/10.1007/s10295015-1682-x.

12. Olano C, Lombó F, Méndez C, Salas J. Improving production of bioactive secondary metabolites in actinomycetes by metabolic engineering. Metab Eng. 2008;10(5):281-92. https://doi.org/10.1016/j.ymben.2008.07.001.

13. Chappuis F, Sundar S, Hailu A, Ghalib H, Rijal S, Peeling R, et al. Visceral leishmaniasis: what are the needs for diagnosis, treatment and control? Nat Rev Microbiol. 2007;5(S11):S7-16. https://doi.org/10.1038/nrmicro1748z.

14. Sundar S, Rai M, Chakravarty J, Agarwal D, Agrawal N, Vaillant M, et al. New treatment approach in Indian visceral Leishmaniasis: single-dose liposomal amphotericin B followed by short-course Oral Miltefosine. Clin Infect Dis. 2008;47(8):1000-6. https://doi.org/10.1086/591972.

15. Mathew B, Nath M. Recent approaches to antifungal therapy for invasive mycoses. ChemMedChem. 2009;4(3):310-23. https://doi.org/10.1002/cmdc.2 00800353.

16. Aparicio J, Barreales E, Payero T, Vicente C, de Pedro A, Santos-Aberturas J. Biotechnological production and application of the antibiotic pimaricin: biosynthesis and its regulation. Appl Microbiol Biotechnol. 2015;100(1):6178.

17. Ren J, Cui Y, Zhang F, Cui H, Ni X, Chen F, et al. Enhancement of nystatin production by redirecting precursor fluxes after disruption of the tetramycin gene from Streptomyces ahygroscopicus. Microbiol Res. 2014;169(7-8):602-8. https://doi.org/10.1016/j.micres.2013.09.017.

18. Song $Y, H e ~ L$, Chen $L$, Ren $Y$, Lu H, Geng S, et al. Baseline sensitivity and control efficacy of antibiosis fungicide tetramycin against Botrytis cinerea. Eur J Plant Pathol. 2016;146(2):337-47. https://doi.org/10.1007/s10658-0160920-Z.

19. Gao Y, He L, Li X, Lin J, Mu W, Liu F. Toxicity and biochemical action of the antibiotic fungicide tetramycin on Colletotrichum scovillei. Pestic Biochem Physiol. 2018;147:51-8. https://doi.org/10.1016/j.pestbp.2018.02.012.

20. Ma D, Zhu J, Jiang J, Zhao Y, Li B, Mu W, et al. Evaluation of bioactivity and control efficacy of tetramycin against Corynespora cassiicola. Pestic Biochem Physiol. 2018;152:106-13. https://doi.org/10.1016/j.pestbp.2018.09.009.

21. Radics $L$, Incze $M$, Dornberger $K$, Thrum $H$. Tetramycin $B$, a new polyene macrolide antibiotic. Tetrahedron. 1982;38(1):183-9. https://doi.org/10.1016/ 0040-4020(82)85064-3.

22. Zhang $N$, Song $Z$, Xie $Y$, Cui $P$, Jiang $H$, Yang $T$, et al. Identification and characterization of antifungal active substances of Streptomyces hygroscopicus BS-112. World J Microbiol Biotechnol. 2013;29(8):1443-52. https://doi.org/10.1007/s11274-013-1307-3.

23. Cao B, Yao F, Zheng X, Cui D, Shao Y, Zhu $C$, et al. Genome Mining of the Biosynthetic Gene Cluster of the Polyene macrolide antibiotic Tetramycin and characterization of a P450 Monooxygenase involved in the hydroxylation of the Tetramycin B Polyol segment. ChemBioChem. 2012; 13(15):2234-42. https://doi.org/10.1002/cbic.201200402.

24. Cui H, Ni X, Shao W, Su J, Su J, Ren J, et al. Functional manipulations of the tetramycin positive regulatory gene ttmRIV to enhance the production of tetramycin a and nystatin A1 in Streptomyces ahygroscopicus. J Ind Microbiol Biotechnol. 2015;42(9):1273-82. https://doi.org/10.1007/s10295-015-1660-3.

25. Cui H, Ni X, Liu S, Wang J, Sun Z, Ren J, et al. Characterization of three positive regulators for tetramycin biosynthesis in Streptomyces ahygroscopicus. FEMS Microbiol Lett. 2016;363(12):fnw109.

26. Volokhan O, Sletta H, Ellingsen T, Zotchev S. Characterization of the P450 Monooxygenase NysL, responsible for C-10 hydroxylation during biosynthesis of the Polyene macrolide antibiotic Nystatin in Streptymyces noursei. Appl Environ Microbiol. 2006;72(4):2514-9. https://doi.org/10.1128/A EM.72.4.2514-2519.2006.

27. Byrne B, Carmody M, Gibson E, Rawlings B, Caffrey P. Biosynthesis of Deoxyamphotericins and Deoxyamphoteronolides by engineered strains of 
Streptomyces nodosus. Chem Biol. 2003;10(12):1215-24. https://doi.org/10.1 016/j.chembiol.2003.12.001.

28. Mendes M, Antón N, Martín J, Aparicio J. Characterization of the polyene macrolide P450 epoxidase from Streptomyces natalensis that converts deepoxypimaricin into pimaricin. Biochem J. 2005;386(1):57-62. https://doi. org/10.1042/BJ20040490

29. Huang K, Zhang B, Shen Z, Cai X, Liu Z, Zheng Y. Enhanced amphotericin B production by genetically engineered Streptomyces nodosus. Microbiol Res. 2021;242:126623. https://doi.org/10.1016/j.micres.2020.126623.

30. Kwan D, Schulz F. The stereochemistry of complex Polyketide biosynthesis by modular Polyketide synthases. Molecules. 2011;16(7):6092-115. https:// doi.org/10.3390/molecules16076092.

31. Myronovskyi M, Luzhetskyy A. Native and engineered promoters in natural product discovery. Nat Prod Rep. 2016;33(8):1006-19. https://doi.org/10.103 9/C6NP00002A.

32. Chang $Y$, Chai B, Ding $Y$, He M, Zheng L, Teng $Y$, et al. Overproduction of gentamicin B in industrial strain Micromonospora echinospora CCTCC M 2018898 by cloning of the missing genes genR and genS. Metab Eng Commun. 2019;9:e00096. https://doi.org/10.1016/j.mec.2019.e00096.

33. Tan G, Deng K, Liu X, Tao H, Chang Y, Chen J, et al. Heterologous biosynthesis of Spinosad: an Omics-guided large Polyketide synthase gene cluster reconstitution in Streptomyces. ACS Synth Biol. 2017:6(6):995-1005. https://doi.org/10.1021/acssynbio.6b00330

34. Dhakal D, Le T, Pandey R, Jha A, Gurung R, Parajuli P, et al. Enhanced production of Nargenicin A1 and generation of novel glycosylated derivatives. Appl Biochem Biotechnol. 2015;175(6):2934-49. https://doi.org/1 0.1007/s12010-014-1472-3.

35. Jung W, Yoo Y, Park J, Park S, Han A, Ban Y, et al. A combined approach of classical mutagenesis and rational metabolic engineering improves rapamycin biosynthesis and provides insights into methylmalonyl-CoA precursor supply pathway in Streptomyces hygroscopicus ATCC 29253. App Microbiol Biotechnol. 2011;91(5):1389-97. https://doi.org/10.1007/s00253011-3348-6.

36. Yu Z, Lv H, Wu Y, Wei T, Yang S, Ju D, et al. Enhancement of FK520 production in Streptomyces hygroscopicus by combining traditional mutagenesis with metabolic engineering. Appl Microbiol Biotechnol. 2019; 103(23-24):9593-606. https://doi.org/10.1007/s00253-019-10192-8.

37. Yi J, Kim M, Kim E, Kim B. Production of pikromycin using branched chain amino acid catabolism in Streptomyces venezuelae ATCC 15439. J Ind Microbiol Biotechnol. 2018;45(5):293-303. https://doi.org/10.1007/s10295-01 8-2024-6.

38. Beites T, Rodríguez-García A, Moradas-Ferreira P, Aparicio J, Mendes M. Genome-wide analysis of the regulation of pimaricin production in Streptomyces natalensis by reactive oxygen species. Appl Microbiol Biotechnol. 2014;98(5):2231-41. https://doi.org/10.1007/s00253-013-5455-z.

39. Santos-Aberturas J, Engel J, Dickerhoff J, Dörr M, Rudroff F, Weisz K, et al. Exploration of the substrate promiscuity of biosynthetic tailoring enzymes as a new source of structural diversity for Polyene macrolide antifungals. ChemCatChem. 2014;7(3):490-500.

\section{Publisher's Note}

Springer Nature remains neutral with regard to jurisdictional claims in published maps and institutional affiliations.

Ready to submit your research? Choose BMC and benefit from:

- fast, convenient online submission

- thorough peer review by experienced researchers in your field

- rapid publication on acceptance

- support for research data, including large and complex data types

- gold Open Access which fosters wider collaboration and increased citations

- maximum visibility for your research: over $100 \mathrm{M}$ website views per year

At $\mathrm{BMC}$, research is always in progress.

Learn more biomedcentral.com/submissions 